\title{
Assessment of Applicability of Portable HPGe Detector with In Situ Object Counting System based on Performance Evaluation of Thyroid Radiobioassays
}

\author{
MinSeok Park, Tae-Eun Kwon, Min Jung Pak, Se-Young Park, Wi-Ho Ha*, Young-Woo Jin \\ National Radiation Emergency Medical Center, Korea Institute of Radiological and Medical Sciences, Seoul, Korea
}

\section{Original Research}

Received December 19, 2016

Revision March 30, 2017

Accepted May 15, 2017

Corresponding author:Wi-Ho Ha

National Radiation Emergency Medical Center, Korea Institute of Radiological and Medical Science, 75 Nowon-gil, Nowongu, Seoul 01812, Korea

Tel: $+82-2-3399-5961$

Fax: +82-2-3399-5960

E-mail: lovin@kirams.re.kr

This is an Open-Access article distributed under the terms of the Creative Commons Attribution NonCommercial License (http://creativecommons.org/ licenses/by-nc/4.0) which permits unrestricted noncommercial use, distribution, and reproduction in any medium, provided the original work is properly cited.

Copyright $\odot$ 2017 The Korean Association for Radiation Protection

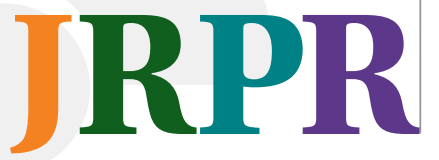

Background: Different cases exist in the measurement of thyroid radiobioassays owing to the individual characteristics of the subjects, especially the potential variation in the counting efficiency. An In situ Object Counting System (ISOCS) was developed to perform an efficiency calibration based on the Monte Carlo calculation, as an alternative to conventional calibration methods. The purpose of this study is to evaluate the applicability of ISOCS to thyroid radiobioassays by comparison with a conventional thyroid monitoring system.

Materials and Methods: The efficiency calibration of a portable high-purity germanium (HPGe) detector was performed using ISOCS software. In contrast, the conventional efficiency calibration, which needed a radioactive material, was applied to a scintillator-based thyroid monitor. Four radioiodine samples that contained ${ }^{125} \mathrm{I}$ and ${ }^{131} \mathrm{I}$ in both aqueous solution and gel forms were measured to evaluate radioactivity in the thyroid. ANSI/HPS N13.30 performance criteria, which included the relative bias, relative precision, and root-mean-squared error, were applied to evaluate the performance of the measurement system.

Results and Discussion: The portable HPGe detector could measure both radioiodines with ISOCS but the thyroid monitor could not measure ${ }^{125} \mathrm{I}$ because of the limited energy resolution of the $\mathrm{NaI}(\mathrm{Tl})$ scintillator. The ${ }^{131} \mathrm{I}$ results from both detectors agreed to within $5 \%$ with the certified results. Moreover, the ${ }^{125}$ I results from the portable HPGe detector agreed to within $10 \%$ with the certified results. All measurement results complied with the ANSI/HPS N13.30 performance criteria.

Conclusion: The results of the intercomparison program indicated the feasibility of applying ISOCS software to direct thyroid radiobioassays. The portable HPGe detector with ISOCS software can provide the convenience of efficiency calibration and higher energy resolution for identifying photopeaks, compared with a conventional thyroid monitor with a $\mathrm{NaI}(\mathrm{Tl})$ scintillator. The application of ISOCS software in a radiation emergency can improve the response in terms of internal contamination monitoring.

Keywords: Radiation emergency, Radioactivity, Radioiodine, Monte Carlo method, Radiobioassay

\section{Introduction}

Radioactive sources are widely used in nuclear power, nuclear medicine, radiopharmaceutical production, and research laboratories. However, their use can lead to health problems in occupational workers and the public via radiation or radionuclides entering the body through various routes such as inhalation, ingestion, skin absorption, and wound absorption [1-6]. Thus, it is necessary to prepare appropriate mea- 
surement methods and equipment for assessing the internal radiation dose in case of a radiation accident [6]. In particular, radioiodine is commonly used for medical purposes, and easily induces internal contamination owing to its volatility at room temperature [7]. Moreover, large amounts of radioiodine are produced in nuclear power plants, and can be a major cause of radioactive contamination by being released into the atmosphere in the event of a nuclear accident. The International Commission on Radiological Protection (ICRP) reported that $30 \%$ of radioiodine intake was accumulated in the thyroid gland and the remainder was excreted through urine [4]. In addition, the average excess relative risk of radiation-induced thyroid cancer was $1.5 \mathrm{~Sv}^{-1}$ (all ages and genders) from the life span study in the United Nations Scientific Committee on the Effects of Atomic Radiation (UNSCEAR) report [8]. Therefore, it is necessary to evaluate the internal deposition of radioiodine to monitor internal exposure.

Internal contamination in the thyroid gland is generally detected using a thyroid monitoring system. Typically, thyroid monitoring involves a type of gamma spectroscopy that measures gamma-emitting radionuclides in the sample $[1$, 9]. A typical thyroid monitor for radiobioassays is a detector with $\mathrm{NaI}(\mathrm{Tl})$ scintillation material. The installation cost of a $\mathrm{NaI}(\mathrm{Tl})$-based monitoring system is not prohibitive, and a $\mathrm{NaI}(\mathrm{Tl})$ scintillator has high sensitivity to gamma radiation. However, $\mathrm{NaI}(\mathrm{Tl})$ scintillators provide low energy resolution when compared with semiconductor detectors. Moreover, $\mathrm{NaI}(\mathrm{Tl})$ scintillators require a sealed assembly to protect the hygroscopic material from moisture in the air.

Recently, semiconductor materials such as high-purity germanium (HPGe) have been used in in-vivo measurement devices for internal dose assessment. HPGe detectors show high performance when measuring radionuclides in bioassay samples, and have a high energy resolution, despite a major disadvantage of requiring a cooling system [10]. This technical advancement means that the amount of the radioactive source inside a human body can be estimated by utilizing a semiconductor detection system, according to the invivo radiobioassay method. In the National Radiation Emergency Medical Center (NREMC) of the Korea Institute of Radiological and Medical Sciences (KIRAMS), various types of radiation monitoring systems have been used for evaluating internal contamination, such as the scintillator-based thyroid monitor and portable HPGe detector with the In situ Object Counting System (ISOCS). The portable HPGe detector with the ISOCS, developed by Canberra Industries, is commonly used as a field-based detection system to measure a contaminated material or area. To respond to a radiation emergency adequately, it is necessary to manage the performance of the monitoring systems to evaluate the contamination levels and internal dose accurately [11]. To test the performance of the thyroid monitoring systems, we participated in the intercomparison program provided by Lawrence Livermore National Laboratory (LLNL). In this study, ISOCS was applied to perform the thyroid radiobioassay for confirming the feasibility of alternative methodologies for efficiency calibration in the thyroid radiobioassay.

\section{Materials and Methods}

\section{LLNL Thyroid Radioiodine Intercomparison program} The Thyroid Radioiodine Intercomparison program (TRIP) arranged by the LLNL was performed to assess thyroid radiobioassays and validate measurement results in respect of the measured activity of radioiodine samples. This program was established to evaluate the performance of radioiodine measurements and to ensure regulation compliance. The TRIP also provided the International Atomic Energy Agency (IAEA)/American National Standards Institute (ANSI) neck calibration phantom [12] in addition to National Institute of Standards and Technology (NIST) traceable isotope samples of ${ }^{125} \mathrm{I}$ and ${ }^{131} \mathrm{I}$.

\section{Radioiodine samples and detection system}

The TRIP provided six radioiodine samples in $30 \mathrm{~mL}$ vials. As shown in Figure 1, three samples (vials A, B, and C) contained an aqueous solution of $30 \mathrm{~mL}$, and the other vials (vials $\mathrm{D}, \mathrm{E}$, and F) contained a gel of $20 \mathrm{~mL}$ The aqueous solutions contained radioiodine (A: ${ }^{125} \mathrm{I}, \mathrm{B}:{ }^{131} \mathrm{I}$, and C: control sample) comprised of sodium iodide $(100 \mathrm{mg} / \mathrm{mL})$ and sodium thiosulfate $(200 \mathrm{mg} / \mathrm{mL})$. The gel type samples contained the same material (D: ${ }^{125} \mathrm{I}, \mathrm{E}:{ }^{131} \mathrm{I}$, and F: iodine carrier as a control sample) as aqueous solution, but were mixed with approximately $400 \mathrm{mg}$ of SP400 waterworks crystals. We transferred aqueous solution samples to $20 \mathrm{~mL}$ polyethylene vials to meet the requirements of our measurement geometry based on the efficiency calibration data of the retained monitoring system. The gel-type samples could not be transferred to the $20 \mathrm{~mL}$ vials owing to the viscosity of the gel material.

Two types of detection systems operated in NREMC were used in the current intercomparison program. One was a portable HPGe detector (Falcon 5000 Radionuclide Identifi- 

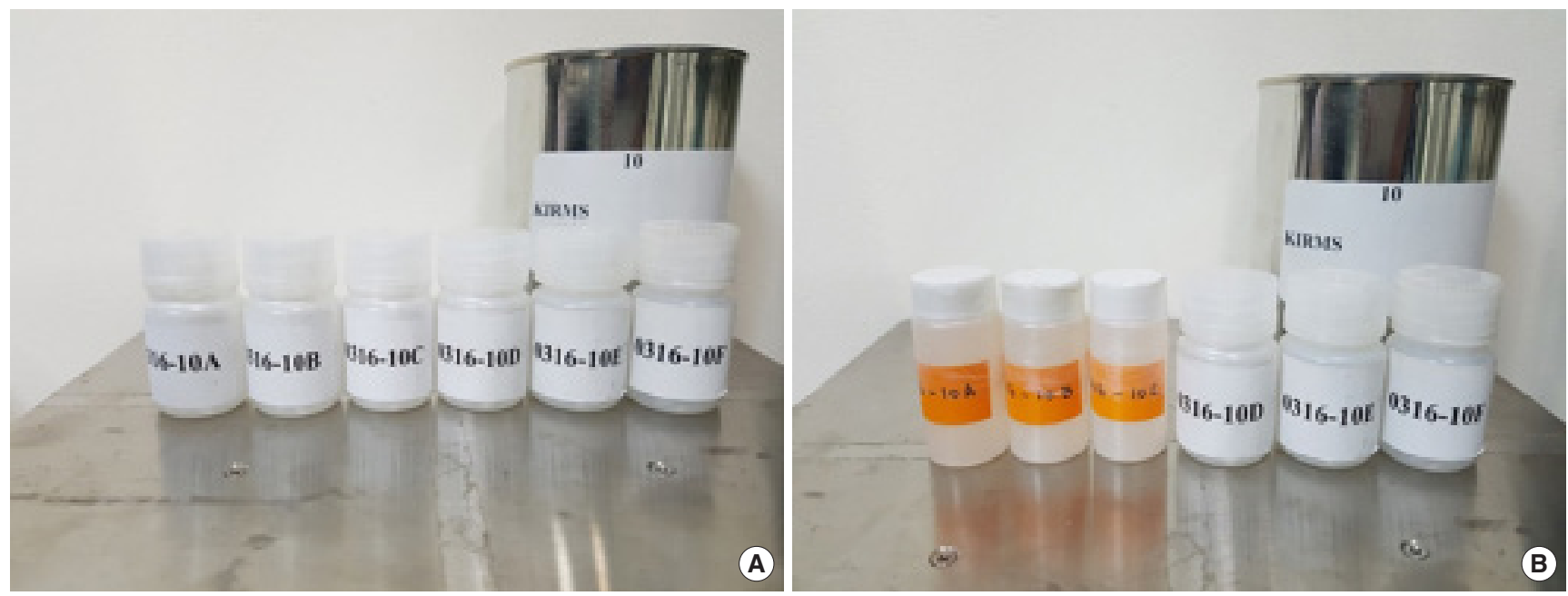

Fig. 1. Radioiodine samples for the intercomparison program (A) Samples provided by the LLNL, (B) Samples modified for measurement.

Detection System

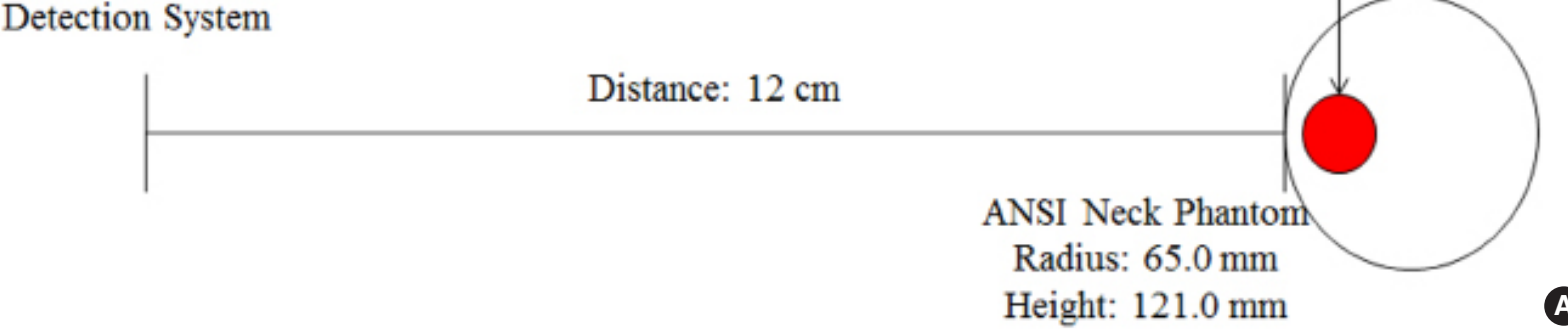

Radioactive Source

- Radius: $13.0 \mathrm{~mm}$

- Height: $41.0 \mathrm{~mm}$

Radioactive Source

- Radius: $17.0 \mathrm{~mm}$

- Height: $25.0 \mathrm{~mm}$

Detector System

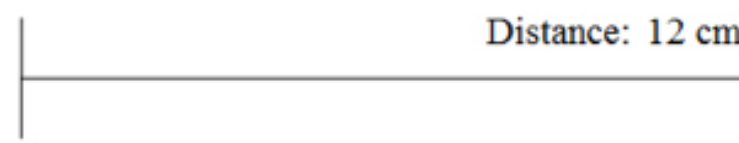

ANSI Neck Phantom

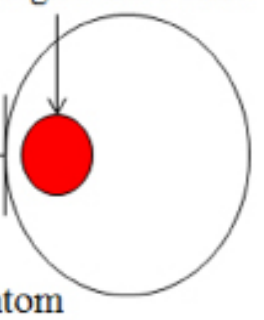

- Radius: $65.0 \mathrm{~mm}$

- Height: $121.0 \mathrm{~mm}$

B

Fig. 2. Schematic of the geometry for measuring radioiodine samples (A) Aqueous solution samples, (B) Gel samples.

er, Canberra Industries, Meriden, CT) with a broad-energy germanium (BEGe) semiconductor sensor, and the other was a thyroid monitor (802, Canberra Industries, Meriden, $\mathrm{CT})$ with a $2 \times 2 \mathrm{in}$. $\mathrm{NaI}(\mathrm{Tl})$ scintillator. These systems were controlled by using the GENIE-2000 software (Canberra In- dustries, Meriden, CT). BEGe, a type of the HPGe, provides enhanced efficiency and energy resolution at the low-gamma-energy range.

The measurement geometry is shown in Figure 2. The distance between the detector and ANSI neck calibration phan- 
tom was $12 \mathrm{~cm}$. The standard neck phantom was mostly used for the calibration and measurement to simulate the attenuation of the overlying tissue thickness [13]. The vial containing the radioactive source was placed in the hole of the neck phantom. The difference in the sample size (height and radius) was an important point to be considered, with respect to the calibration geometry. In this intercomparison program, only the aqueous solution samples agreed with the efficiency calibration data that we had previously calculated, owing to the measurement geometry and certified radionuclide material. Therefore, the additional calibration by the ISOCS and Monte Carlo methods with MCNPX 2.7.0 (Los Alamos National Laboratory, NM) was applied to samples A, $\mathrm{D}$, and $\mathrm{E}$ in consideration of the measurement geometry and emitted energy of ${ }^{125} \mathrm{I}$.

The energy calibration procedure was performed using a mixed gamma ray reference material in $22 \mathrm{~mL}$ vials. The calibrated energies for the portable HPGe detector and thyroid monitor ranged from 59.5 to $1,836.0 \mathrm{keV}$. The low-energy calibration below $59.5 \mathrm{keV}$ was performed by using a correlation equation of energies with the detector channel.

The conventional efficiency calibration was implemented in the thyroid monitor. By using the thyroid monitor, the ${ }^{131} \mathrm{I}$ sample in the $20 \mathrm{~mL}$ vial could be measured, but samples $\mathrm{D}$ and $\mathrm{E}$ could not be measured owing to the limitation on the measurable energy range of the thyroid monitor. The geltype sample could not be measured directly because of the geometrical discrepancy with the reference radioactive material, but ${ }^{131}$ I gel type sample was measured by applying an efficiency correction factor calculated by Monte Carlo simulation. MCNPX software was implemented to obtain the factor for correcting the geometrical discrepancy.

Efficiency calibration of the portable HPGe detector was performed using the ISOCS software. The geometry was composed and the efficiency calibration data were calculated by considering the calibration condition of each sample. The ISOCS was developed by Canberra Industries for efficiency calibration of the detection systems. The calibration was performed by using the Geometry Composer feature and the Monte Carlo radiation transport method. Geometry Composer is a support program in the ISOCS software for imitating various types of measurement samples. Based on the simulation geometry replicated by the geometry composer software, the counting efficiency could be calculated by using the ISOCS software. Thus, ISOCS provides convenient efficiency calibration with the capability to measure radioactive samples that have different shapes, sizes, chemical compositions, material fill-height, and wall materials. Radioactive sources in arbitrary containers can be measured by using the ISOCS modeling technique, without any calibration standard or radioactive reference materials. In conventional calibration, it is necessary to perform efficiency calibration with standardized measurement geometry and radioactive reference materials. Therefore, the ISOCS software provides a cost-effective method for radionuclide verification.

\section{Calculation and evaluation techniques}

The number of net counts measured was converted to activity in accordance with IAEA TECDOC-1401 [14]. This report presented the factors related with the characteristics of radionuclides and measurement conditions that are used for calculating the activities of sources. The equation for calculating the activity is given below:

$$
A=\frac{N}{\varepsilon \times I_{\gamma} \times t_{s} \times K_{l}}
$$

where $\mathrm{A}$ is the activity $(\mathrm{Bq})$ of the sample, $\varepsilon$ is the efficiency at the photo-peak energy, $I_{\gamma}$ is the branching ratio of the gamma radiation, $t_{s}$ is the live time(s) of the count collection, and $K_{1}$ is the correction factor for the nuclide decay from the time the sample was collected to the start of spectrum acquisition. Photopeaks of $364.4 \mathrm{keV}$ and $35.5 \mathrm{keV}$ were used to estimate the activity of ${ }^{131} \mathrm{I}$ and ${ }^{125} \mathrm{I}$ samples, respectively. The live time for sample measurement was $240 \mathrm{~s}$, according to the time of a routine thyroid examination in NREMC. The activities extracted from the results of five repeated measurements of each sample were evaluated with respect to the ANSI/HPS N13.30 performance requirements (1996 and 2011) for relative bias and relative precision. ANSI/HPS N13.30-1996 recommended that the performance criterion of relative bias was a measurement range from $-25 \%$ to $+50 \%$ of the reference measurement result, and the performance criterion of relative precision was lower than $40 \%$ [15]. The ANSI/HPS N13.30 report described the relative bias and relative precision given by the following equations [15-17]:

$$
\begin{gathered}
B_{r i}=\frac{A_{i}-A_{a i}}{A_{a i}} \\
-0.25 \leq B_{r}=\sum_{i=1}^{N} \frac{B_{r i}}{N} \leq 0.50
\end{gathered}
$$

and 


$$
S_{B}=\sqrt{\frac{\sum_{i=1}^{B}\left(B_{r i}-B_{r}\right)^{2}}{N-1}} \leq 0.40
$$

where $B_{r i}$ is the relative bias for the $\mathrm{i}_{\text {th }}$ measurement in each sample, $A_{i}$ is the activity measured in NREMC, $A_{a i}$ is the result measured by the host institute (LLNL), $B_{r}$ is the average relative bias for a minimum of five measurements, and $S_{B}$ is the relative precision, which represents the relative dispersion of the values from their average. $\mathrm{N}$ is the number of sample measurements, which should be at least five. The performance criteria for a radiobioassay were evaluated by using the root mean squared error (RMSE). The RMSE test results guarantee the performance of a detection system and ensure nuclear analytical compliance with ANSI/HPS N13.30 report-2011. The performance criterion for radiobioassays in ANSI/HPS 13.30-2011 was suggested as RMSE $\leq$ $25 \%$. Based on the relative bias and precision, the RMSE was calculated as [16]:

$$
R M S E=\sqrt{B_{r}^{2}+S_{B}^{2}} \leq 0.25
$$

\section{Results and Discussion}

The comparison of efficiency calibration curves between the thyroid monitor and portable HPGe detector with the ISOCS for the $20 \mathrm{~mL}$ aqueous solution of ${ }^{131} \mathrm{I}$ is shown in Figure 3. The efficiency calibration curve is the curve of fitted values of the measured efficiency for each emission peak. A polynomial equation, usually used in efficiency fitting for cal-

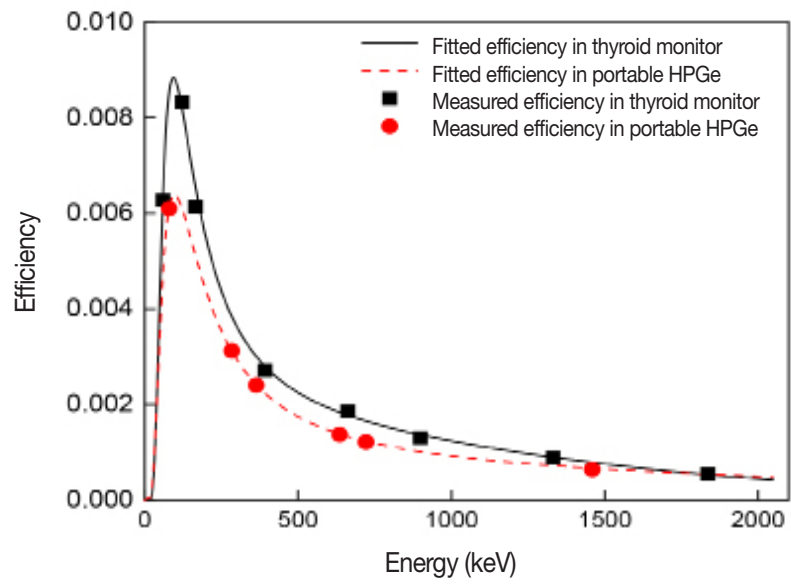

Fig. 3. Efficiency calibration curves of the thyroid monitor and portable HPGe detector with the ISOCS $\left({ }^{131}\right.$ I) for $20 \mathrm{~mL}$ aqueous solution samples. culating the fitted efficiency, was applied for fitting the efficiency curve, based on the software algorithm. The average relative deviation between fitted efficiency and measured efficiency in the thyroid monitor was $2.61 \%$. The efficiency calibration curve of the thyroid monitor was the conventional efficiency calibration data that covered an energy range of 59.51,836.0 keV from the reference radioactive material. For the portable HPGe detector, the efficiency calibration data were calculated with the ISOCS software using only the ${ }^{131}$ I aqueous solution sample for the intercomparison measurement. The average relative deviation between fitted and measured efficiency was within $1 \%$ in the ISOCS efficiency calibration. By using the ISOCS software, it was possible to calibrate the detection system without using reference radionuclide materials. The points of measured efficiency in the curve were the emission peaks of ${ }^{131} \mathrm{I}$. The counting efficiency of the thyroid monitor was generally higher than that of the portable HPGe detector owing to the high intrinsic efficiency of the $\mathrm{NaI}(\mathrm{Tl})$ scintillation material. At $364.4 \mathrm{keV}$, which was the peak energy with the highest branching ratio for ${ }^{131} \mathrm{I}$, the calculated efficiency of the thyroid monitor and portable HPGe detector were 0.00294 with a full width at half maximum (FWHM) of $31.4 \mathrm{keV}$, and 0.00241 with a FWHM of $1.97 \mathrm{keV}$, respectively. The efficiency of the portable HPGe detector at $364.4 \mathrm{keV}$ was $18.0 \%$ lower than that of the thyroid monitor.

Figure 4 shows the sample activity measured by the portable HPGe and thyroid monitor. The ${ }^{125} \mathrm{I}$ in samples A and D could not be measured by the thyroid monitor because of the limit of energy resolution of $\mathrm{NaI}(\mathrm{Tl})$. The activity results of samples were about $3.85 \%$ higher than the certified activi-

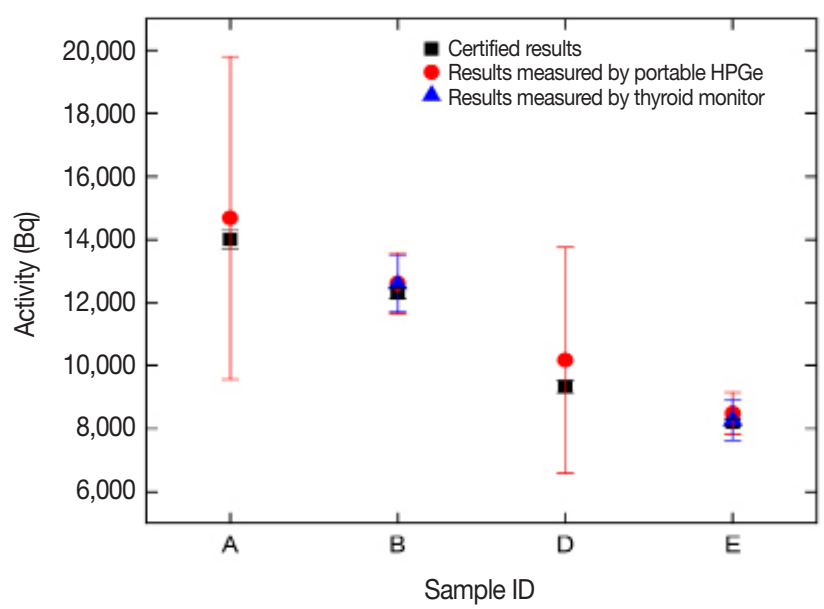

Fig. 4. Comparison of the sample activity results obtained with the portable HPGe detector and thyroid monitor. 
Table 1. Activities of Radioiodine Samples and Performance Evaluation Results Obtained with the Portable HPGe Detector

\begin{tabular}{|c|c|c|c|c|c|c|c|c|c|c|}
\hline \multirow[b]{2}{*}{ Sample ID } & \multirow[b]{2}{*}{ Nuclide } & \multicolumn{2}{|c|}{ Certified Value } & \multicolumn{2}{|c|}{ Reported Value } & \multicolumn{5}{|c|}{ Performance Evaluation } \\
\hline & & $\begin{array}{c}\text { Activity } \\
\text { (Bq) }\end{array}$ & $\begin{array}{c}\text { Relative } \\
\text { expanded } \\
\text { uncertainty } \\
(\%, \mathrm{k}=3)\end{array}$ & $\begin{array}{c}\text { Averaged } \\
\text { activity } \\
(\mathrm{Bq}, \mathrm{N}=5)\end{array}$ & $\begin{array}{c}\text { Relative } \\
\text { expanded } \\
\text { uncertainty } \\
(\%, \mathrm{k}=3)\end{array}$ & $\begin{array}{c}\text { Relative } \\
\text { bias }\end{array}$ & $\begin{array}{l}\text { Relative } \\
\text { precision }\end{array}$ & RMSE & $\begin{array}{c}\text { Meets ANSI } \\
13.30 \\
-1996\end{array}$ & $\begin{array}{c}\text { Meets ANSI } \\
13.30 \\
-2011\end{array}$ \\
\hline A & $\mathrm{I}-125$ & 14,000 & 2.0 & 14,683 & 34.9 & 0.049 & 0.037 & 0.061 & yes & yes \\
\hline B & $\mid-131$ & 12,300 & 1.4 & 12,615 & 7.7 & 0.026 & 0.012 & 0.028 & yes & yes \\
\hline D & $\mid-125$ & 9,330 & 2.0 & 10,171 & 35.2 & 0.090 & 0.037 & 0.097 & yes & yes \\
\hline E & $\mid-131$ & 8,200 & 1.4 & 8,488 & 7.8 & 0.035 & 0.018 & 0.039 & yes & yes \\
\hline
\end{tabular}

ty results from the host institute. In the case of the portable HPGe detector, the efficiency calibration was performed by using the Monte Carlo method of ISOCS. Compared with the experimental efficiency, it was possible to calculate an underestimated efficiency by using ISOCS because the ISOCS calculated the efficiency under ideal conditions with an ideal background radiation. In the conventional efficiency calibration, the difference in radioactive material could affect the efficiency calibration. The composition and size of radioactive material (22 mL, BACTO Agar) used for efficiency calibration differed from those of radioiodine samples $(20 \mathrm{~mL}$, solution and gel type) provided by the host institute. Practically, it is very difficult to manufacture radioactive material customized with characteristics of samples to be tested to reduce the possible error that arises from the composition and size of the respective samples. After considering several factors that can lead to erroneous measurement results, the optimized radioactive materials were used for the calibration of the detection system.

The difference between the activities of the ${ }^{125}$ I samples (A and D) was greater than that corresponding to ${ }^{131} \mathrm{I}$ samples (B and $\mathrm{E}$ ). When measuring the activity of the ${ }^{125} \mathrm{I}$ sample, it was difficult to calibrate the detection system and measure the sample activity because of its low gamma emission energy and emission rate; ${ }^{125}$ I emitted $35.4 \mathrm{keV}$ gamma rays with a $6.68 \%$ branching ratio.

In general, the efficiency is measured by using the reference radioactive material, which has an energy range between $59.5\left({ }^{241} \mathrm{Am}\right)$ and 1,836.0 $\left({ }^{88} \mathrm{Y}\right) \mathrm{keV}$. However, it is difficult to measure the efficiencies in low energy (below about $45 \mathrm{keV}$ ) using a source-based calibration, because of the lack of calibration sources or X-ray radiation emitted from the shield [18]. Thus, it is possible to miscalculate the efficiency under the minimum energy of the reference radioactive material. However, efficiencies can be acquired by using the ISOCS software at any measurable energy range of the detec- tor. The activity results of samples A and D measured with the portable HPGe detector demonstrated that the efficiency obtained by the ISOCS software was reliable.

More details on the measured values and performance evaluation of the portable HPGe measurement system are given in Table 1. As given in Table 1, the portable HPGe detector could measure the ${ }^{125}$ I radioisotope that emitted 35.4$\mathrm{keV}$ gamma rays, because of its broad energy range. ${ }^{131} \mathrm{I}$ radioisotopes were measured with both detection systems; however, ${ }^{125}$ I radioisotopes could only be measured by the portable HPGe detector owing to the limited performance inherent in $\mathrm{NaI}(\mathrm{Tl})$ scintillators. Activities of samples A and D that contained ${ }^{125}$ I were $14,683 \pm 5,138 \mathrm{~Bq}$ (relative expanded uncertainty: $34.9 \%$ ) and 10,171 $\pm 3,587 \mathrm{~Bq}$ (relative expanded uncertainty: $35.2 \%$ ), respectively. This relative expanded uncertainty represents an interval having a confidence level greater than $99 \%$ by applying a coverage factor of $k=3$. The relative bias and relative precision between the reported and certified values were 0.049 and 0.037 for sample A, and 0.090 and 0.037 for sample $\mathrm{D}$, respectively. Based on these evaluation factors, the RMSE values of samples A and D were 0.061 and 0.097 , respectively.

The measured activities of the ${ }^{131}$ I radionuclide in samples $\mathrm{B}$ and $\mathrm{E}$ were $12,615 \pm 972 \mathrm{~Bq}$ (relative expanded uncertainty: $7.7 \%$ ) and $8,488 \pm 663 \mathrm{~Bq}$ (relative expanded uncertainty: $7.8 \%$ ), respectively. The relative bias and relative precision of samples B and E were less than half those of samples A and D. The reason for such differences of performance evaluation factors is the gamma emission rate and photo-peak energy of ${ }^{125} \mathrm{I}$ (35.4 keV, 6.68\% branching ratio) and ${ }^{131} \mathrm{I}$ ( $364 \mathrm{keV}$, $81.2 \%$ branching ratio) radionuclides. Samples B and E had RMSE values of 0.028 and 0.039 , respectively. As shown in the performance evaluation results, the values measured by the portable HPGe were generally in agreement with the reference values with respect to the relative bias and RMSE. Percentages of relative bias in all samples indicated less than 
Table 2. Activities of Radioiodine Samples and Performance Evaluation Results Obtained with the Thyroid Monitor

\begin{tabular}{|c|c|c|c|c|c|c|c|c|c|c|}
\hline \multirow[b]{2}{*}{ Sample ID } & \multirow[b]{2}{*}{ Nuclide } & \multicolumn{2}{|c|}{ Certified Value } & \multicolumn{2}{|c|}{ Reported Value } & \multicolumn{5}{|c|}{ Performance Evaluation } \\
\hline & & $\begin{array}{l}\text { Activity } \\
\text { (Bq) }\end{array}$ & $\begin{array}{c}\text { Relative } \\
\text { expanded } \\
\text { uncertainty } \\
(\%, \mathrm{k}=3)\end{array}$ & $\begin{array}{l}\text { Averaged } \\
\text { activity } \\
(\mathrm{Bq}, \mathrm{N}=5)\end{array}$ & $\begin{array}{c}\text { Relative } \\
\text { expanded } \\
\text { uncertainty } \\
(\%, \mathrm{k}=3)\end{array}$ & $\begin{array}{c}\text { Relative } \\
\text { bias }\end{array}$ & $\begin{array}{l}\text { Relative } \\
\text { precision }\end{array}$ & RMSE & $\begin{array}{c}\text { Meets ANSI } \\
13.30 \\
-1996\end{array}$ & $\begin{array}{c}\text { Meets ANSI } \\
13.30 \\
-2011\end{array}$ \\
\hline B & $\mid-131$ & 12,300 & 1.4 & 12,587 & 7.1 & 0.023 & 0.027 & 0.036 & yes & yes \\
\hline$E$ & $\mid-131$ & 8,200 & 1.4 & 8,266 & 7.7 & 0.008 & 0.016 & 0.018 & yes & yes \\
\hline & & & & $7,706^{\star}$ & 7.7 & 0.060 & 0.068 & 0.091 & yes & yes \\
\hline
\end{tabular}

${ }^{*}$ There were results applied to no efficiency correction depending on the difference of sample geometry.

$10 \%$ difference. The RMSE values of each sample were lower than 0.25 and thus met the performance criteria of ANSI/ HPS 13.30-2011. The sample activities measured by the portable HPGe detector fulfilled the performance criteria recommended by ANSI/HPS 13.30-1996.

Table 2 shows the values measured by the thyroid monitor and performance evaluation results. The reported activity of sample B was $12,587 \pm 887 \mathrm{~Bq}$, with a relative expanded uncertainty of $7.1 \%$. The evaluation result of the measured values represented a relative bias of 0.023 and a relative precision of 0.027 . The RMSE of this sample was 0.036 , which met the performance criterion. Two measured values of sample $\mathrm{E}$ are given in the table, owing to the difference in efficiency depending on the sample geometry. In conventional efficiency calibration, it was necessary to reconcile the calibration geometry and measurement geometry to reduce the counting error. Hence, the correction factor obtained from the Monte Carlo simulation was applied to the measurement data of sample E to reduce the counting error caused by the geometry. The activity of sample E with correction was $8,266 \pm 634 \mathrm{~Bq}$, with a relative expanded uncertainty of $7.7 \%$, and the activity with no correction result was 7,706 $\pm 591 \mathrm{~Bq}$. The activity with correction was closer to the certified value. Moreover, the relative bias, relative precision, and RMSE also decreased after efficiency correction by the Monte Carlo technique. However, the relative precision of the thyroid monitor was larger than its relative bias, which was different from the results of the portable HPGe detector. The signal response of $\mathrm{NaI}(\mathrm{Tl})$ is less repeatable than that of HPGe, because $\mathrm{NaI}(\mathrm{Tl})$ has the detection mechanism to convert the light induced by incident radiation in the scintillation material into a signal by using a photomultiplier tube and light sensor. In addition, $\mathrm{NaI}(\mathrm{Tl})$ can be easily influenced by the environmental factors such as moisture in air and background radiation. For this reason, the measured activities by the thyroid monitor were dispersed from their average. All of the measurement results fulfilled the performance criteria of both ANSI/HPS-1996 and 2011. This means that the differences in the geometry of the source material rarely affect the measurement results. According to other studies, the most important factor that could influence the measurement data in thyroid monitoring was the overlying tissue thickness in front of the thyroid gland $[13,19]$. Although more accurate measurement results could be acquired by applying the proper efficiency correction, measurement with a small difference in geometry, excluding the overlying tissue thickness, would have acceptable quality for thyroid monitoring during a radiation emergency.

The relative bias of the portable HPGe detector was under $4 \%$ and $9 \%$ in ${ }^{131} \mathrm{I}$ and ${ }^{125} \mathrm{I}$, respectively. In the case of the thyroid monitor, the relative bias was under $3 \%$ in ${ }^{131}$ I with an efficiency correction by the Monte Carlo method (under 6\% without correction). Considering the relative bias of the portable HPGe detector and thyroid monitor, all the measurement results showed good agreement with the internationally certified value (less than $10 \%$ of the relative bias). Moreover, all of the measurement data represented acceptable results in accordance with the ANSI/HPS reports. This demonstrates that measurements with the ISOCS software are technically feasible for thyroid radiobioassays.

\section{Conclusion}

This study presents the intercomparison results from participation in the TRIP. The data measured by the portable HPGe detector and $\mathrm{NaI}(\mathrm{Tl})$ thyroid monitor agreed well with the certified result, but the portable HPGe detector with ISOCS software provided convenient efficiency calibration and the ability to detect radionuclides over a wide range of gamma energies for thyroid monitoring. Based on the evaluation results of the thyroid monitoring, we concluded that the portable HPGe detector with ISOCS software would be 
applicable for the estimation of radioiodine intake. Although this study only provided results related to thyroid monitoring, further research on applicable measurement techniques with the ISOCS software would be desirable for use in radiation emergency-response situations.

\section{Acknowledgements}

This study was supported by a grant of the Korea Institute of Radiological and Medical Sciences (KIRAMS), funded by Ministry of Science, ICT and Future Planning, Republic of Korea (1711031804/50445-2016). The authors would like to appreciate the Lawrence Livermore National Laboratory (LLNL) for providing the opportunity to participate in the intercomparison program on thyroid radiobioassay.

\section{References}

1. Dantas BM, Lucena EA, Dantas ALA, Santos MMS, Juliao LQC, Melo DR, Sousa WO, Fernandes PC, Mesquita SA. A mobile bioassay laboratory for the assessment of internal doses based on in vivo and in vitro measurement. Health Phys. 2010;99:449-452.

2. Yoo JR, Park SY, Yoon SY, Ha WH, Lee SS, Kim KP. Radiobioassay performance evaluation of urine and faeces samples for radiation emergency preparedness. J. Nucl. Sci. Technol. 2016;53(11): 1742-1748.

3. International Commission on Radiological Protection. Dose coefficients for intakes of radionuclides by workers. ICRP publication 68. 1994;1-17.

4. International Commission on Radiological Protection. Individual monitoring for internal exposure of workers. ICRP publication 78. 1997;3-18.

5. National Council on Radiation Protection and Measurements. Use of bioassay procedures for assessment of internal radionuclide deposition. NCRP report no. 87. 1987;19-31.

6. International Atomic Energy Agency. Indirect methods for assessing intake of radionuclides causing occupational exposure. Safety reports series no. 18. 2000;15-21.

7. Canada Nuclear Safety Commission. Thyroid screening for ra- dioiodine. Regulatory document no. 58. 2008;1-15.

8. United Nations Scientific Committee on the Effects of Atomic Radiation. Sources, Effects, and Risks of Ionization Radiation. UNSCEAR 2000 report. 2000;338-342.

9. Bento J, Martins B, Teles P, Neves M, Colarinha P, Alves F, Teixeira N, Vaz P, Zankl M. Performance assessment and uncertainty evaluation of a portable NaI-based detection system used for thyroid monitoring Radiat. Prot. Dosim. 2012;151:252-261.

10. Fantinova K, Fojtik P. Monte Carlo simulation of the BEGe detector response function for in vivo measurements of 241Am in the skull. Radiat. Phys. Chem. 2014;104:345-350.

11. National Council on Radiation Protection and Measurements. Management of persons accidentally contaminated with radionuclides. NCRP report no. 65. 1979;20-44.

12. International Commission on Radiation Units and Measurements. Phantoms and computational models in therapy, diagnosis and protection. ICRU report no. 48. 1992;31, 98.

13. Ghare VP, Patni HK, Akar DK, Rao DD. Counting efficiency of whole-body monitoring system using BOMAB and ANSI/IAEA thyroid phantom due to internal contamination of I-131. Radiat. Prot. Dosim. 2014;162:230-235.

14. International Atomic Energy Agency. Quantifying uncertainty in nuclear analytical measurements. IAEA-TECDOC-1401. 2004; 103-126.

15. Health Physics Society. Performance criteria for radiobioassay. ANSI/HPS N.13.30-1996. American National Standards Institute. 1996;23-29.

16. Health Physics Society. Performance criteria for radiobioassay. ANSI/HPS N.13.30-2011. American National Standards Institute. 2011;6-12

17. International Organization for Standardization. Radiation protection - performance criteria for radiobioassay: ISO 28218. 2010; 7-12.

18. Morris KE, Mueller WF, Blanc P, Bronson F, Croft S, Field MB, Nakazawa DR, Venkataraman R, Zhu H. The efficiency characterization of Germanium detectors at energies less than $45 \mathrm{keV}$. Waste Management Conference. Phoenix, AZ. March 7-11, 2012.

19. Damet J, Bochud FO, Bailat C, Laedermann JP, Baechler S. Variability of radioiodine measurements in the thyroid. Radiat. Prot. Dosim. 2010;144:326-329. 